\title{
Evaluation of the effect of anti-pollution \& anti-aging eye cream on the collagen contraction
}

\author{
Gülşah Gedik ${ }^{1 *}$, Seda Alaca² \\ ${ }^{1}$ Department of Pharmaceutical Technology, Faculty of Pharmacy, Trakya University, Edirne, Turkey \\ ${ }^{2}$ Tan-Alize Cosmetics, İstanbul, Turkey
}

\begin{tabular}{|c|c|}
\hline \multicolumn{2}{|c|}{ ARTICLE INFO } \\
\hline \multicolumn{2}{|c|}{ Article history: } \\
\hline Received & 24 May 2020 \\
\hline Revised & 28 May 2020 \\
\hline Accepted & 31 May 2020 \\
\hline Online & 01 Jun 2020 \\
\hline Published & 15 Jun 2020 \\
\hline \multicolumn{2}{|l|}{ Keywords: } \\
\hline \multicolumn{2}{|l|}{ Anti-aging } \\
\hline \multicolumn{2}{|c|}{ Anti-pollution } \\
\hline \multicolumn{2}{|l|}{ Collagen } \\
\hline \multicolumn{2}{|l|}{ Eye cream } \\
\hline \multicolumn{2}{|c|}{ Hydrolyzed algin } \\
\hline $\begin{array}{l}{ }^{*} \text { Correspon } \\
\text { gulsahged }\end{array}$ & $\begin{array}{l}\text { ing author: } \\
\text { @trakya.edu.tr }\end{array}$ \\
\hline
\end{tabular}

\begin{abstract}
A B S T R A C T
This is a study of the brown algae-derived hydrolyzed algin as used in eye cream formulation. Alginic acid sodium is a gelling and non-toxic anionic polysaccharide, which is used to bone tissue engineering, preparation of alginate hydrogels, encapsulating, hydrating, protective, and de-polluting action. The skin of eye contour is thinner and less dense in support fibres than other parts of the face. Therefore, cutaneous sagging is frequently seen to appear around 40 years of age, very often aggravated by environmental factors such as pollution. The objective of the study was to evaluate the effect of anti-pollution \& anti-aging eye cream on the collagen contraction. The outcomes of the characterization analysis indicate the development of successful cream formulation with optimum characteristics. No microbial growth was observed. The collagen lattices were prepared with human dermal fibroblasts, for evaluation of the effect of the cream on the collagen contraction. The lattices were treated or not (control) with the tested cream and then incubated at $37^{\circ} \mathrm{C}$ for 96 hours. The surface of lattices was measured by image analysis, and the lattices contraction was analyzed at 16, 24, 40, 48, 64, 72, 88, and 96 hours (the measure of lattices area). The treatment with eye cream at $0.5 \%$ decreases the surface of the lattice compared to the control (no treatment). According to the results obtained under the conditions of the test, the eye cream tends to increase the collagen contraction and a significative decrease of the lattice surface by $6.68 \%$ compared to the control at 16 hours.
\end{abstract}

This is an open-access article licensed under the Creative Commons Attribution 4.0 International License (CC-BY). (cc) BY

\section{INTRODUCTION}

This is a study of the brown algae-derived hydrolyzed algin as used in cosmetics. The ingredient in this study is the extract of the whole or a defined part of the seaweed. "Brown algae" is a common name for seaweeds of the class Phaeophyceae and classified in about 265 genera with more than 1500 species [1,2].

The actual color varies depending on the proportion of brown pigment (fucoxanthin) to green pigment (chlorophyll). This algal group contains alginic acid and fucoidan in its complex cell walls. Several brown algae constituents, such as phytosterols, phytosteryl ingredients, and alginic acid, were found to be safe $[3,4]$. The most frequently reported function of brown algae in cosmetics is as a skin-conditioning agent; other reported functions include absorbent, antioxidant, binder, hair conditioning agent, oxidizing agent, and viscosity increasing agent [4].

Alginic acid sodium is a gelling and non-toxic anionic polysaccharide. The carboxylic acid groups on the alginic acid chain render it insoluble in water. However, converting alginic acid to its sodium form enables it to solubilize in water easily [5].
Hydrolyzed algin is used:

- in combination with chitosan, to fabricate a biodegradable porous scaffold for bone tissue engineering [6],

- to study the characteristics of a modified amphiphilic alginate derivative [7],

- to the study the impact of alginate on the rate of lipid digestion by employing an in vitro digestion model [8],

- in the preparation of alginate hydrogels [9],

- as an encapsulating agent of $\beta$-galactosidase microparticles $[10]$

- as an occlusive and hydrating agent,

- for protective and de-polluting action [11].

Brown algae have been used for wastewater/effluent treatment and removal of heavy metals (Sargassum, Laminaria, and Ecklonia species) [12].

Alginates, which are membrane polysaccharides taken from the skin of algae, are depolymerized to obtain a high molecular weight oligoalginate. Applied to the skin, their high degree of polymerization enables them to stay on the surface of the epidermis and form a protective mask.

Every day the skin is exposed to all sorts of impurities in the atmosphere, such as heavy metals, cigarette smoke, and 
so on. These reduce the levels of hydration and oxygenation and produce free radicals, which lead to cutaneous aging. Alginates minimizes the adhesion of chelates heavy metals such as lead and cadmium to stop particles from encrusting and asphyxiating the skin. Alginates also protects the viability of cells exposed to cigarette smoke [13].

The skin of the eye contour covers 22 muscles, which are continually moving. To make this mobility possible, this area is thinner and less dense in support fibres than other parts of the face. Therefore, cutaneous sagging is frequently seen to appear around 40 years of age, very often aggravated by environmental factors such as pollution [14].

The objective of the study was to evaluate the effect of anti-pollution \& anti-aging eye cream on the collagen contraction.

\section{MATERIALS AND METHODS}

\subsection{Materials}

All chemicals were of reagent and analytical grade. Double-distilled water was used throughout the study. All chemicals CAS No and functions were given in Table 1.

Table 1. The complete formula of eye cream formulation

\begin{tabular}{|c|c|c|c|}
\hline \multicolumn{4}{|c|}{ Chemical Analysis Properties } \\
\hline Components & $\% \mathrm{w} / \mathrm{w}$ & CAS No & Function \\
\hline Water & q.s. & $7732-18-5$ & solvent \\
\hline Glycerin & 5.000 & $56-81-5$ & humectant \\
\hline Cetearyl Alcohol & 4.500 & $67762-27-0$ & emulsifying \\
\hline Glyceryl Stearate & & $31566-31-1$ & \\
\hline Ceteareth-20 & $400 \Omega$ & $68439-49-6$ & emollient \\
\hline Ceteareth-12 & 4.000 & $68439-49-6$ & emulsifying \\
\hline Cetyl Palmitate & & $540-10-3$ & \\
\hline $\begin{array}{l}\text { Caprylic/Capric } \\
\text { Triglyceride }\end{array}$ & 2.500 & $73398-61-5$ & skin conditioning \\
\hline $\begin{array}{l}\text { Dicaprylyl } \\
\text { Carbonate }\end{array}$ & 2.000 & $1680-31-5$ & emollient \\
\hline
\end{tabular}

\section{Seawater,}

Hydrolyzed Algin,

Phenethyl Alcohol,

1.500

Sucrose

\begin{tabular}{lccc} 
Phenoxyethanol & 0.765 & $122-99-6$ & preservative skin \\
Ethylhexylglycerin & 0.085 & $70445-33-9$ & conditioning \\
\hline Cera Alba & 0.600 & $8012-89-3$ & emollient \\
\hline Mica & 0.500 & $12001-26-2$ & opacifying \\
\hline $\begin{array}{l}\text { Butyrospermum } \\
\text { Parkii (Shea Butter) }\end{array}$ & 0.500 & $\begin{array}{c}194043-92-0 \\
91080-23-8\end{array}$ & skin conditioning \\
\hline Sodium Polyacrylate & 0.300 & $\begin{array}{c}9003-04-7 \\
25549-84-2\end{array}$ & $\begin{array}{c}\text { emollient } \\
\text { film forming }\end{array}$ \\
\hline Xanthan Gum & 0.050 & $11138-66-2$ & viscosity controlling \\
\hline Tocopheryl Acetate & 0.001 & $\begin{array}{c}7695-91-2 \\
58-95-7\end{array}$ & antioxidant \\
\hline
\end{tabular}

\subsection{Preparation of Eye Cream Formulation}

The complete formula is reflected in Table 1. All the aqueous phase materials and the oil phase ingredients were placed in two separate containers and heated to above $75^{\circ} \mathrm{C}$. The water phase was then added to the oil phase using continuous agitation. Preservative, antioxidant, humectant agents and hydrolyzed algin mixture were added after cooling. The formulation was kept at $25 \pm 1^{\circ} \mathrm{C}$ for 48 hours to see a possible phase separation.

\subsection{Characterization of Formulation}

The formulation was evaluated for its sensorial parameters and physicochemical parameters like $\mathrm{pH}$, density, and viscosity. The sensorial parameters of the formulation, such as appearance, odor, color, were determined. The $\mathrm{pH}$ of the formulation was detected by a digital $\mathrm{pH}$-meter (Mettler Toledo S 220, Switzerland), the density was detected by a pycnometer (Mettler Toledo 30330857, Switzerland), and the viscosity measurements were performed with a vibro viscometer (AND, SV-10, Japan). The experiments were repeated five times at $25^{\circ} \mathrm{C}$.

\subsection{Microbial Contamination Tests}

The microbiological contamination of formulations was evaluated by validated test kits from AFNOR using cultures of Staphylococcus aureus, Escherichia coli, Pseudomonas Aeruginosa, total bacteria, yeast, and mold.

\subsection{Evaluation of The Effect of an Eye Cream on The Collagen Contraction}

For technical reasons, the tested formulation was previously extracted. It was first diluted to $10 \%$ in the medium; the insoluble mixture was stirred for 1 hour to extract the active ingredients. After centrifugation, the supernatant containing the active principles (aqueous phase) was collected and diluted in culture medium at specified concentrations. This study was done with the support of ARERKO.

\subsubsection{Biological model}

The collagen lattice model was used, which corresponds to a three dimensional (3D) reconstituted dermis, with a population of fibroblasts synthesizing new fibers and interacting with collagen fibers network (confidential composition).

\subsubsection{Preparation of the lattices}

The cells were Normal human dermal fibroblasts from a donor of 30 years old, passage 12 .

The collagen lattices were prepared with these fibroblasts in $5 \%$ of serum. The lattices contraction was analyzed after $16,24,40,48,64,72,88$, and 96 hours of culture (photographs and measure of lattices area).

\subsubsection{Treatment}

The lattices were treated or not (control) with the tested cream and then incubated at $37^{\circ} \mathrm{C}$ for 96 hours. The lattices contraction was analyzed at 16, 24, 40, 48, 64, 72, 88, and 96 hours (measure of lattices area). The surfaces of lattices were measured by image analysis at a different time of incubation $(16,24,40,48,64,72,88$, and 96 hours).

\subsubsection{Data expression}

Results were expressed as the percentage of the surface after the contraction in the function of the initial surface (measured at $t_{0}$ before incubation). The more the percentage is low, the more the contraction is good. 


\subsection{Statistical Analysis}

The raw data were transferred and processed using MS Excel Software. The different conditions were compared using the Student's t-test. A difference between the two groups was considered statistically significant if the $p$-value was less than 0.05 , which was noted $* p<0.05$. If the $p$-value is lower than 0.01 and 0.001 , it was noted $* * p<0.01$ and $* * * \mathrm{p}<0.001$, respectively.

\section{RESULTS AND DISCUSSION}

The physicochemical and sensorial characterization parameters of the formulation are reported in Table 2. The density, $\mathrm{pH}$, and viscosity of all formulations were found to be satisfactory. The $\mathrm{pH}$ of the developed cream ranged between 5.5-6.0. The density of the developed cream found $0.98 \pm 0.02 \mathrm{~g} / \mathrm{mL}$. The viscosity found $14.10 \pm 0.22 \mathrm{P}$. The density, $\mathrm{pH}$, and viscosity of the cream was appropriate for the dermal application.

Table 2. The physicochemical and sensorial characterization parameters of the eye cream formulation

\begin{tabular}{lc}
\hline & Physicochemical Parameters \\
\hline Density $(\mathrm{g} / \mathrm{mL})$ & $0.98 \pm 0.02\left(25^{\circ} \mathrm{C}\right)$ \\
$\mathrm{pH}$ & $5.75 \pm 0.10\left(25^{\circ} \mathrm{C}\right)$ \\
Viscosity $(\mathrm{P})$ & $14.10 \pm 0.22\left(25^{\circ} \mathrm{C}\right)$ \\
\hline \multicolumn{2}{c}{ Sensorial Parameters } \\
\hline Appearance & Cream \\
Odor & Characteristic \\
Color & White \\
\hline
\end{tabular}

\subsection{Microbial Contamination Tests}

All results about microbiological contamination studies were given in Table 3. After the incubation period, the tests were checked for microbial growth. No microbial growth was observed. The obtained results had confirmed the microbial study of our formulation.

Table 3. The microbiological study results

\begin{tabular}{lc}
\hline Test Microorganisms & Microbiological Parameters \\
\hline Total Bacteria & $<100 \mathrm{CFU} / \mathrm{mL}$ \\
Yeast and Mould & - \\
Escherichia coli & - \\
Staphylococcus aureus & - \\
Pseudomonas aeruginosa & - \\
\hline
\end{tabular}

\subsection{Evaluation of The Effect of an Eye Cream on The Collagen Contraction}

The treatment with eye cream (118-08) at $0.5 \%$ decrease the lattice surface compared to the control (no treatment). The cream visually decreased the lattice surface over time, which means an increase in the collagen contraction (Figure 1-3).

\subsection{Discussion}

This study evaluated an eye cream on the collagen contraction in vitro in human dermal fibroblasts cultured in a lattice of collagen that appeared to be a model close to the living dermis. Today, the use of extracts as anti-pollutant and anti-aging from various plants and marine organisms has become popular. The objective of the study was to evaluate the effect of anti-pollution \& anti-aging eye cream on the collagen contraction.

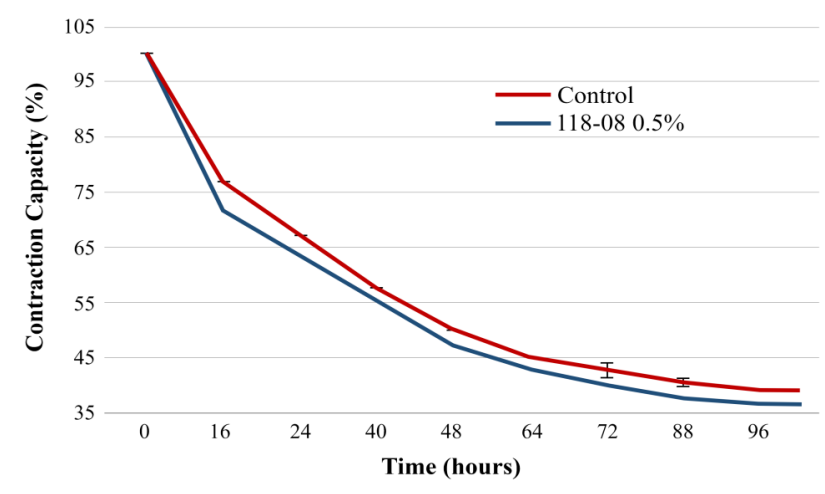

Figure 1. Effect of the eye cream (118-08) at $0.5 \%$ on the lattices surface over time

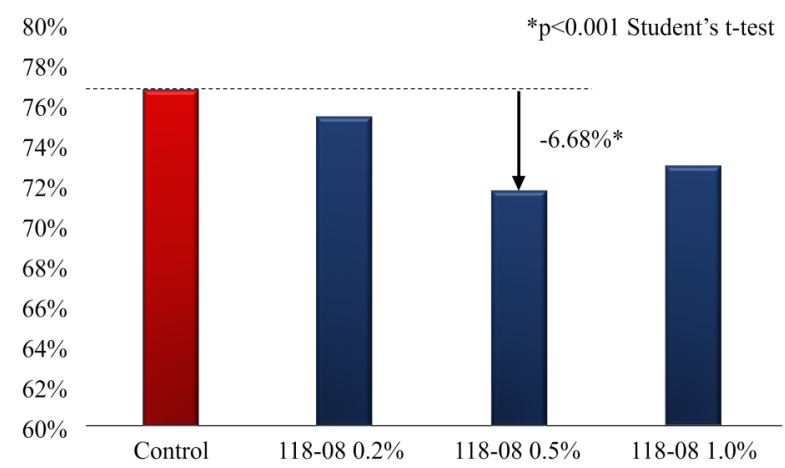

Figure 2. Effect of the eye cream (118-08) at $0.5 \%$ on the lattice surface at 16 hours
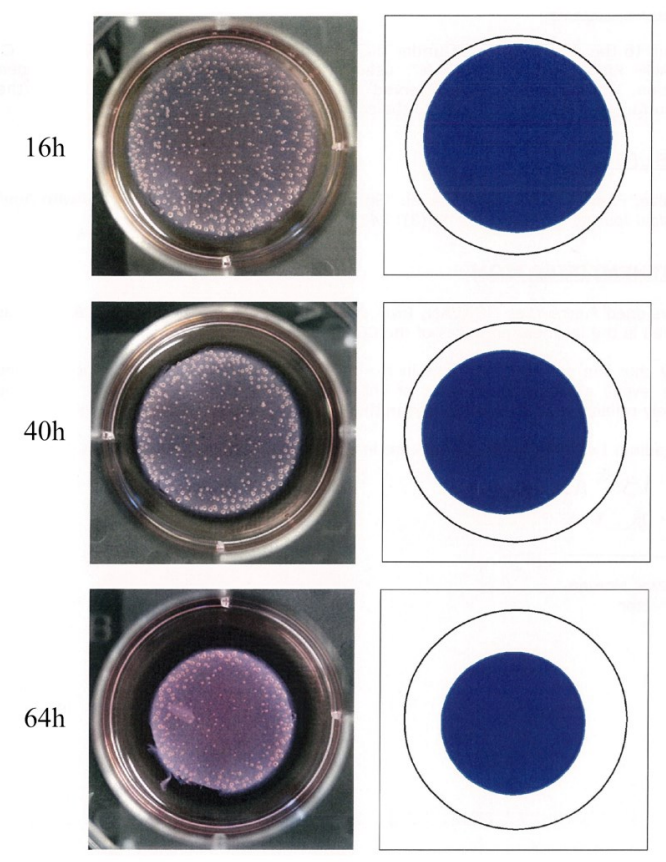

Figure 3. Observation of one lattice treated with the eye cream (118-08) at 0.5\% (16, 40, and 64 hours)

Environmental pollutants have a negative effect on human health and human skin. Exposure to pollutants can cause aging, pigmentation, acne, atopic dermatitis, psoriasis, and even skin cancer, or acne [15]. 
Pollution has a negative impact that can be observed at the stratum corneum, which is generally colonized with residual microorganisms. In the presence of pollutants, the skin microbiome changes for the benefit of pathogenic bacteria [16]. Moreover, contamination enhances the production of reactive oxygen species and causes disturbance in the redox balance. Some pollutants also tend to permeate through the stratum corneum into deeper skin layers. There, they act as a ligand for the aryl hydrocarbon receptor (AhR), which takes part in mediating the toxic effects of pollutants. All of them cause the induction of an inflammatory cascade in the skin. The increased production of pro-inflammatory cytokines, such as interleukin $1 \beta$ or interleukin 8 , greatly impacts the biological function of the cells, resulting in skin lesions and deterioration of skin appearance $[17,18]$.

Vierkötter et al. showed a direct link between chronic exposure to traffic-related particulate matter and the occurrence of prominent skin aging signs, especially pigment spots, but also wrinkles in Caucasian women [19].

$\mathrm{Li}$ et al. then reported epidemiological evidence that indoor air pollution from cooking with solids fuels was associated with wrinkles in Chinese women [20]. A recent study has found that exposure to $\mathrm{NO}_{2}$ was associated with the formation of lentigines in Caucasian and East Asians [21].

Particles can serve as carriers for organic chemicals and metals that are capable of localizing in mitochondria and generating ROS directly in mitochondria leading to collagen degradation in human skin and thereby cause wrinkle formation $[22,23]$.

In recent years, there has been an increasing interest in products that protect us from the negative impact of pollutants, and that helps to restore the skin barrier function.

The cream was made for this purpose, and depolymerized oligoalginate of high molecular weight was used as an active agent. Applied on human skin, it protects against heavy metals, particles, air pollution, and cigarette smokes. Alginate chelate metal ions, reduce inflammatory mediators, and cytokines. Shanura et al. showed alginic acid also reduced the levels of COX-2, interleukin 6 , TNF- $\alpha$, and inhibited specific key molecular mediators of the NF- $\kappa \mathrm{B}$ and MAPK pathways in keratinocytes. Alginic acid substantially reduced the levels of metal ions like $\mathrm{Pb} 2+$ and $\mathrm{Ca} 2+$ in keratinocytes attributable to its metal ion chelating properties. These cells presented with increased levels of NO, iNOS, COX-2, PGE2, and proinflammatory cytokines [24].

The cream formulation characterized based on its $\mathrm{pH}$, density, and viscosity. Physicochemical characterization of the formulation is an important subject to be considered in the formulation part, especially those intended for dermal application. The cream has good appearance, color, and odor on sensorial inspection. The $\mathrm{pH}$ of the developed cream ranged between 5.5 and 6.0. Ideally, dermal formulations should possess $\mathrm{pH}$ in the range of 5.0-6.0. The basic formulation does not include the hydrolyzed algin, which served as the control for density analysis, presented a density value of $1.01 \mathrm{~g} / \mathrm{mL}$, which is similar to the values registered for the remaining formulation that $0.98 \mathrm{~g} / \mathrm{mL}$. The hydrolyzed algin incorporated into the eye cream formulation did not affect the density of the basic formulation.
Microbial contamination study is crucial to evaluate the microbial stability of formulation to ensure its safety. No microbial growth was observed for the eye cream formulation.

Cellular stiffness is significantly increased in dermal fibroblasts during aging in vivo. This increase in stiffness has a direct impact on cellular contraction capacity.

Human dermal fibroblasts cultured in a lattice of collagen appeared to be a model close to the living dermis. The collagen polymerizes into anchoring fibrils known to bind to cells. Then, the fibroblasts pull on these fibrils thanks to their migratory movement and thus reorganize the matrix.

The lattice decreases its diameter, which results in a phenomenon called contraction or retraction, indicative of the contractile activity of the fibroblasts [25].

The treatment with eye cream at $0.5 \%$ decreases the lattice surface compared to the control (no treatment). According to the results obtained under the conditions of the test, the eye cream used at $0.5 \%$ tends to increase the collagen contraction. The best results are observed at $0.5 \%$ with a significative decrease of the lattice surface by $6.68 \%$ compared to the control at 16 hours. The cream visually decreased the lattice surface over time, which means an increase in the collagen contraction (Figure 1-3). Our results are compatible with the literature. Park et al. suggested that these alginate oligosaccharides might have the potential to prevent skin aging by promoting collagen synthesis through the inhibition of collagen degrading enzyme [26].

\section{CONCLUSION}

Our study showed that the eye cream formulation obtained from hydrolyzed algin called alginic acid is a potent anti-pollution \& anti-aging agent and can be used in patients with lentigines and pigment spots and wrinkles. Future controlled clinical trials are needed to evaluate the efficacy of the eye cream.

In particular, the search for anti-pollution \& anti-aging agents of natural origin is progressing rapidly, which points to the need for further studies exploring the utilization of the therapeutic agents from Brown algae.

\section{AUTHOR CONTRIBUTIONS}

Concept: GG, SA; Design: GG, SA; Supervision: GG; Materials: SA; Data Collection and/or Processing: GG, SA; Analysis and/or Interpretation: GG; Literature Search: GG; Writing: GG; Critical Reviews: GG, SA.

\section{ACKNOWLEDGMENTS}

The authors declared that this study had received no financial support.

\section{CONFLICT OF INTEREST DECLARATION}

The authors report no conflict of interest. The authors alone are responsible for the content and the writing of the paper.

\section{REFERENCES}

[1] Becker L., Safety assessment of brown algae-derived ingredients as used in cosmetics. Draft Report for Panel Review. (2018); 21-22

[2] Bhutia TK, Petruzzello M, Pallardy R, Rimsa C. Brown Algae. Encyclopaedia Britannica. (2018). Retrieved October 3, 2018 from https://www.britannica.com/science/brownalgae 
[3] Becker LC, Bergfeld WF, Belsito DV. Safety assessment of phytosterols as used in cosmetics. Washington, DC: Cosmetic Ingredient Review (CIR). (2014). Retrieved July 23, 2018 from http://online.personalcarecouncil.org/ctfa-static/online/lists/cirpdfs/ FR651.pdf

[4] Johnson Jr WJ, Heldreth B, Bergfeld WF, Belsito DV, Hill RA, Klaassen CD, Liebler DC, Marks Jr JG, Shank RC, Slaga TJ, Snyder PW, Gill LJ. Safety assessment of polysaccharide gums as used in cosmetics. Washington, DC: Cosmetic Ingredient Review (CIR). (2015). Retrieved July 23, 2018 from https://www.cir-safety.org/ingredients

[5] Teegarden DM. Polymer Chemistry: Introduction to an indispensable science. National Science Teachers Association. Virginia: NSTA Press; (2004). 286 p.

ISBN:9-780-8735-5221-9

[6] Zhensheng L, Hassna R, Kip DH, Demin X, Miqin Z. Chitosanalginate hybrid scaffolds for bone tissue engineering. Biomaterials. (2005); 26(18): 3919-3928.

https://doi.org/10.1016/j.biomaterials.2004.09.062

[7] Broderick E, Lyons H, Pembroke T, Byrne H, Murray B, Hall M. The characterisation of a novel, covalently modified, amphiphilic alginate derivative, which retains gelling and non-toxic properties. J Coll int Science. (2006); 298(1): 154-161.

https://doi.org/10.1016/j.jcis.2005.12.026

[8] Hu M, Li y, Decker EA, McClements DJ. Role of calcium and calcium-binding agents on the lipase digestibility of emulsified lipids using an in vitro digestion model. Food Hydrocoll. (2010); 24(8), 719-772.

https://doi.org/10.1016/j.foodhyd.2010.03.010

[9] Vu TT, Carine Lim C, Lim M. Characterization of leukemic cell behaviors in a soft marrow mimetic alginate hydrogel. $J$ Biomed Mater Res B. (2012); 100(7): 1980-1988. https://doi.org/10.1002/jbm.b.32765

[10] Estevinho BN, Damas AM, Martins P, RochaF. Microencapsulation of $\beta$-galactosidase with different biopolymers by a spray-drying process. Food Res Int. (2014); 64, 134-140. https://doi.org/10.1016/j.foodres.2014.05.057

[11] McHugh DJ. A guide to the seaweed industry. Rome: FAO; (2003). 118 p. ISBN:92-5-104958-0

[12] Davis TA, Volesky B, Mucci A. A review of the biochemistry of heavy metal biosoption by brown algae. Water Res. (2003); 37(18): 4311-4330.

https://doi.org/10.1016/S0043-1354(03)00293-8

[13] Besada V, Andrade J, Schultze F, Gonzales JJ. Heavy metals in edible seaweeds commercialised for human consumption. J Mar Sys. (2018); 75(1-2): 305-313. https://doi.org/10.1016/j.jmarsys.2008.10.010

[14] Cityguard (2018); Retrieved May 05, 2020 from http://www.codiftn.com/en/principesactifs

[15] English JSC, Dawe RS, Ferguson J. Environmental effects and skin disease. Br Med Bull. (2003); 68 (1):129-142. https://doi.org/10.1093/bmb/ldg026
[16] Jo JH, Kennedy EA, Kong HH. Topographical and physiological differences of the skin mycobiome in health and disease. Virulence. (2017); 8(3): 324-333. https://doi.org/10.1080/21505594.2016.1249093

[17] Mancebo SE, Wang SQ. Recognizing the impact of ambient air pollution on skin health. J Eur Acad Dermatol Venereol. (2015); 29 (12): 2326-2332. https://doi.org/10.1111/jdv.13250

18] Rembiesa J, Ruzgas T, Engblom J, Holefors A. The impact of pollution on skin and proper efficacy testing for anti-pollution claims. Cosmetics. (2018); 5(1):4. https://doi.org/10.3390/cosmetics5010004

[19] Vierkötter A, Schikowski T, Ranft U, Sugiri D, Matsui M, Krämer U, Krutmann J. Airborne particle exposure and extrinsic skin aging. $J$ Invest Derm. (2010); 130(12): 2719-2726. https://doi.org/10.1038/jid.2010.204

[20] Li M, Vierkötter A, Schikowski T, Hüls A, Ding A, Matsui MS, Deng B, Ma C, Ren A, Zhang J, Tan J9, Yang Y, Jin L, Krutmann J, Li Z, Wang S. Epidemiological evidence that indoor air pollution from cooking with solid fuels accelerates skin aging in Chinese women. $J$ Derm Sci. (2015); 79(2): 148-154. https://doi.org/10.1016/j.jdermsci.2015.04.001

[21] Hüls A, Vierkötter A, Gao W, Krämer U, Yang Y, Ding A, Stolz S, Matsui M, Kan H, Wang S, Jin L, Krutmann J, Schikowski T. Trafficrelated air pollution contributes to development of facial lentigines: further epidemiological evidence from Caucasians and Asians. $J$ Invest Derm. (2016); 136(5): 1053-1056. https://doi.org/10.1016/j.jid.2015.12.045

[22] Ding A, Yang Y, Zhao Z, Hüls A, Vierkötter A, Yuan Z, Cai J, Zhang J, Gao W, Li J, Zhang M, Matsui M, Krutmann J, Kan H, Schikowski T, Jin L, Wang S. Indoor PM2.5 exposure afects skin aging manifestation in a Chinese population. Sci Rep. (2017); 7: 15329. https://doi.org/10.1038/s41598-017-15295-8

[23] Li N, Sioutas C, Cho A, Schmitz D, Misra C, Sempf J, Wang M, Oberley T, Froines J, Nel A. Ultrafine particulate pollutants induce oxidative stress and mitochondrial damage. Env Health Perspect. (2003); 111(4):455-460. https://doi.org/10.1289/ehp.6000

24] Fernando IPS, Jayawardena TU, Sanjeewa KKA, Wang L, Jeon YJ, Lee WW. Anti-inflammatory potential of alginic acid from Sargassum horneri against urban aerosol-induced inflammatory responses in keratinocytes and macrophages. Ecotoxicol Environ Saf. (2018); 160:24-31 https://doi.org/10.1016/j.ecoenv.2018.05.024

[25] Schulze C, Wetzel F, Kueper T, Anke Malsen A, Muhr G, Jaspers S Blatt T, Wittern KP, Wenck H, Käs JA. Stiffening of human skin fibroblasts with age. Biophys J. (2010); 99(8): 2434-2442. https://doi.org/10.1016/j.bpj.2010.08.026

[26] Park RM, Ji Ahn JY, Kim SY, Wee JH, Kim YH, Jiho Min J. Effect of alginate oligosaccharides on collagen expression in HS 27 human dermal fibroblasts. J Toxicol Environ Health Sci. (2019); 11: 327-334. https://doi.org/10.1007/s13530-019-0421-5 